\title{
Trombosis de la vena dorsal del pene (Flebitis de Mondor). Aportación de un nuevo caso
}

\author{
Ó. Rodríguez Faba*, L. Parra Muntaner**, S.C. Gómez Cisneros**, J.L. Martín Benito*, \\ S. Escaf Barmadah*
}

*Servicio de Urología. Hospital Central de Asturias. Oviedo (Asturias). **Servicio de Urología. Hospital del Bierzo. Ponferrada (León).

Actas Urol Esp 2006; 30 (1): 80-82

\section{RESUMEN}

TROMBOSIS DE LA VENA DORSAL DEL PENE (FLEBITIS DE MONDOR). APORTACIÓN DE UN NUEVO CASO

Objetivo: Presentamos un nuevo caso de trombosis de la vena dorsal superficial del pene o "flebitis de Mondor". Revisamos las características de la enfermedad y los métodos diagnósticos y terapéuticos actuales.

Métodos: Revisamos el caso de un varón de 41 años que consultó por dolor e induración dorsal del pene.

Resultados: Después de realizar exploración física y Eco-doppler fue diagnosticado de Trombosis de la vena dorsal superficial o flebitis de Mondor. Recibió tratamiento con antiinflamatorios no esteroideos y antibióticos experimentando mejoría clínica.

Conclusiones: La trombosis de la vena dorsal del pene es una rara entidad que cursa con dolor e induración dorsal, la etiología puede ser traumática, neoplásica, por excesiva actividad sexual o prolongada abstinencia. Puede cursar de forma aguda, subaguda o crónica. Es necesario realizar diagnóstico diferencial con la linfangitis esclerosante, el estudio de imagen ideal es el Eco-doppler y el tratamiento se basa en antiinflamatorios y antibióticos en caso de infección. Pueden ser útiles sustancias heparinizantes locales reservándose el manejo quirúrgico con trombectomía o resección de la vena dorsal para casos persistentes.

Palabras clave: Trombosis. Mondor. Flebitis.

\section{ABSTRACT \\ TROMBOSIS OF THE DORSAL PENIS VEIN (OF MONDOR'S PHLEBITIS). PRESENTATION OF A NEW CASE}

Objective: We present a new case of trombosis of the superficial dorsal penis vein called Penile Mondor's disease. The characteristics of the disease are reviewed and the most usual diagnostic and therapeutic methods.

Methods: The case of a 41 year old man is reviewed who consulted for pain and induration on the proximal part of the penis.

Results: After phisical examination and Eco-doppler was made the diagnosis of Mondor's disease. He receibed treatment with non steroidal antiinflamatories and antibiotics.

Conclusions: The dorsal vein thrombosis is a rare disease with pain an induration of the dorsal part of the penis. The ethiology can be traumatic, neoplasic, excesive sexual activity or abstinence. Is necesary the diferencial diagnosis with esclerosant linphangitis and the most important imaging is the Eco-doppler. The treatment is based in non steroidal antiinflamatories and antibiotics wit infection. The local aplication of heparine can be useful and the surgery with thrombectomy and resection is for persistent cases.

Keywords: Trombosis. Mondor. Phlebitis. 
$\mathrm{L}$ a trombosis de las venas superficiales de la pared torácica fue descrita por primera vez por Mondor en 1939. Posteriormente BraunFalco describió la flebitis de las venas dorsales del pene como parte de una flebitis generalizada, siendo Helm en 1958 quien publicó la trombosis dorsal aislada de la vena dorsal superficial del pene $^{1}$.

Hasta 1996, 42 casos han sido descritos en la literatura de este proceso, siendo la serie más amplia la presentada por Findlay y Whiting ${ }^{2}$.

El drenaje venoso del pene comienza en la base del glande, varios canales venosos coalescen para formar la vena dorsal del pene, que discurre en un surco entre los cuerpos y drena en el plexo preprostático. Las venas circunflejas se originan en el cuerpo esponjoso y pasan alrededor de los cuerpos cavernosos para reunirse con la vena dorsal profunda perpendicularmente. Ellas sólo están presentes en los dos tercios distales del cuerpo del pene y suman entre tres y diez. Se forman vénulas intermedias de los senos venosos para drenar en un plexo capilar por debajo de la túnica. Estos plexos dan origen a venas emisarias, que por lo común siguen un camino oblicuo entre las capas de la túnica y drenan en las venas circunflejas dorsolateralmente.

Las venas emisarias en el tercio proximal del pene se unen sobre la superficie dorsomedial de los cuerpos cavernosos para formar de dos a cinco venas cavernosas. En el hilio del pene estos vasos pasan entre los pilares y el bulbo y reciben ramas de cada uno de ellos y se unen a las venas pudendas internas.

Este entramado venoso puede verse afectado en procesos inflamatorios ocurridos en determinadas condiciones, como pueden ser traumas sexuales en la parte dorsal y tromboflebitis en la parte ventral ${ }^{2}$.

Presentamos con este artículo un nuevo caso de flebitis de Mondor o trombosis de la vena dorsal superficial revisando los aspectos diagnósticos y terapéuticos recogidos en la literatura.

\section{CASO CLÍNICO}

Presentamos el caso de un paciente de 41 años que consultó por dolor e induración dorsal de pene a nivel del tercio proximal de una semana de evolución. Como antecedente personales, destacaba el ser fumador de 20 cigarrillos al día, y exéresis de quistes en ambas mamas. No existían trastornos de la coagulación ni enfermedades de interés.

El paciente refirió que desde hacía una semana empezó con dolor en el dorso del pene a nivel de la raíz donde palpaba un nódulo indurado y móvil.

A la exploración física los genitales externos eran normales, salvo la palpación dolorosa del nódulo descrito.

Se realizó Ecografía doppler en la que se objetivó una induración dorsal correspondiente a trombosis segmentaria de la vena dorsal superficial del pene siendo permeable el resto de su trayecto (Figs. 1 y 2).

El paciente recibió tratamiento con antiinflamatorios no esteroideos y antibióticos durante cuatro semanas experimentando mejoría clínica del dolor y disminución de la induración.

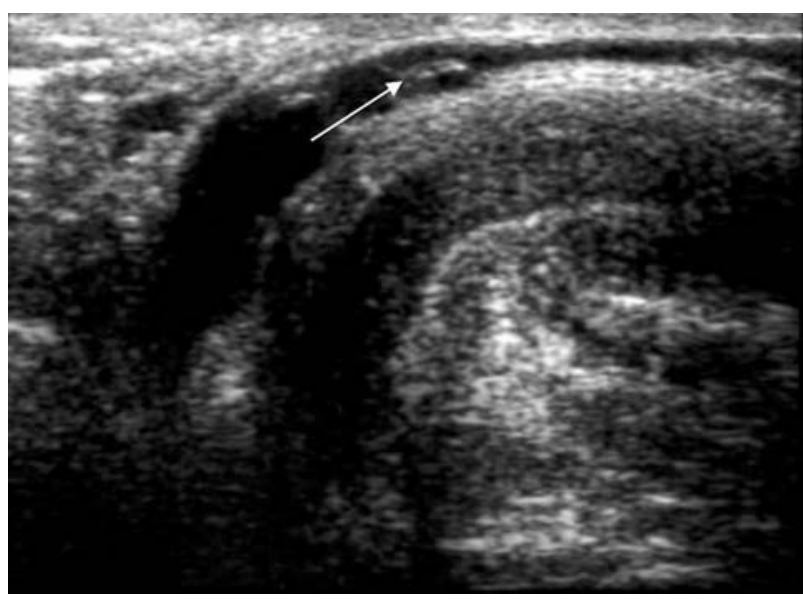

FIGURA 1

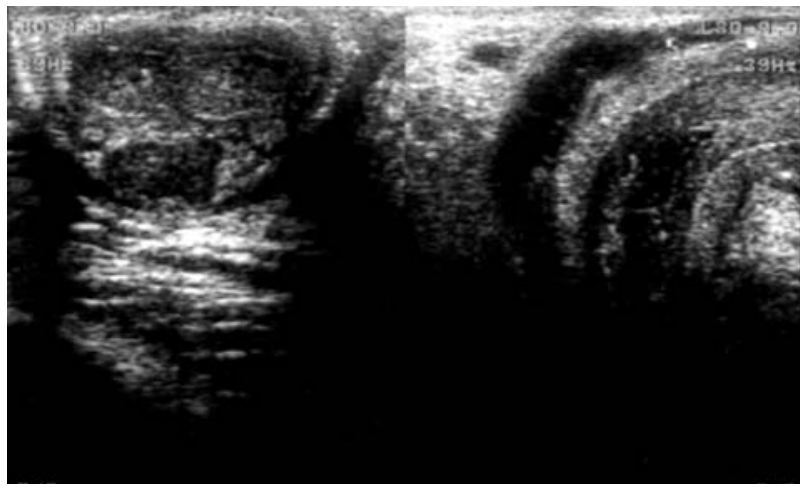

FIGURA 2 
Se solicitó además una resonancia magnética pélvica de control a las seis semanas en la que no se evidenciaron datos de lesión que sugiriesen trombosis u otro tipo de patología en la localización de la vena dorsal de pene.

\section{DISCUSIÓN}

La trombosis de la vena dorsal del pene constituye una rara enfermedad que suele aparecer en hombres entre los 21-70 años ${ }^{1,4}$. Como factores etiológicos están descritos los traumatismos, excesiva actividad sexual, prolongada abstinencia sexual, infecciones locales o a distancia, obstrucción venosa por distensión vesical, tumores pélvicos o elementos constrictores utilizados en determinadas prácticas sexuales, así como el abuso de determinadas drogas endovenosas. Han sido descritas también asociaciones con determinados tumores (vesicales y prostáticos), y un caso de manifestación inicial inusual de adenocarcinoma pancreático diseminado ${ }^{1}$.

La exploración física suele revelar dolor local a la palpación acompañado de una reacción inflamatoria de los tejidos alrededor e induración, en algunos casos los pacientes pueden presentar también síndrome irritativo miccional ${ }^{2,3}$.

La tromboflebitis de la vena dorsal superficial, puede dividirse en tres etapas clínicas:

Aguda, subaguda y recanalizada. La forma aguda suele presentarse en varones entre 20-40 años y suele aparecer en las 24 horas siguientes de una actividad sexual prolongada debido posiblemente a una lesión traumática del endotelio vascular ${ }^{6}$.

El diagnóstico diferencial se plantea con la linfangitis esclerosa del pene, mediante métodos inmunohistoquímicos de determinación de células endoteliales para aclarar el origen venoso y la transiluminación positiva del cordón en la linfangitis $^{2}$.

En cuanto a estudios de imagen, resulta necesario realizar un eco-doppler antes y después del tratamiento ${ }^{5}$. La biopsia y el estudio patológico se realizan de forma infrecuente, resultando una técnica invasiva que no está indicada de rutina ${ }^{2}$.
Existen varias modalidades terapéuticas que deben estar acordes con el estadio clínico de la enfermedad. En principio lo más acertado parece ser una conducta conservadora tranquilizando al paciente y recomendándole reposo sexual hasta la total curación ${ }^{7}$.

La infiltración con anestésicos locales alrededor del segmento venoso afectado, puede ser útil para prevenir el dolor, pero en casos con infección no es efectivo y deben usarse antiinflamatorios y antibióticos. En ocasiones, los procesos agudos han sido tratados con antibióticos y anticoagulantes de forma muy efectiva. Para los estados subagudo y crónico se pueden utilizar antiinflamatorios y cremas locales con heparina.

La mayor parte de los casos se resuelven en 46 semanas, existiendo una recanalización en 9 semanas $^{1-3}$.

En casos persistentes es necesario el manejo quirúrgico con trombectomía o resección de la vena dorsal superficial.

\section{REFERENCIAS}

1. Swierzewski SJ 3rd, Denil J, Ohl DA. The management of penile Mondor's phlebitis: superficial dorsal penile vein thrombosis. J Urol 1993 Jul;150(1):77-78.

2. Sasso F, Gulino G, Basar M, Carbone A, Torricelli P, Alcini E. Penile Mondor`s disease: an underestimated pathology. Br J Urol 1996 May;77(5):729-732.

3. Thomazeau H, Alno L, Lobel B. Thrombosis of the dorsal vein of the penis. A propos of two cases. J Urol (Paris) 1983;89(9):691-692.

4. Ozkara H, Akkus E, Alici B, Akpinar H, Hattat H. Superficial dorsal penile vein thrombosis (penile Mondor's disease). Int Urol Nephrol 1996;28(3):387-391.

5. Le Pionufle N, Djafari M, Garcier JM, De Fraissinette B, Boyer L. Thrombosis of the superficial dorsal vein of the penis (penile Mondor's phlebitis). The interest of Doppler examination). Presse Med 2003 Jun 28;32(23):1074-1076.

6. Arango O, Peyri E, Alvarez-Vijande R. Lesiones vasculares cutáneas de los genitales masculinos. Jarpyo Ed. Madrid 1998;55.

7. Peyri E, Álvarez-Vijande R, Arango O. Enfermedades cutáneas del aparato genital masculino. Monográfico del LXI Congreso Nacional de Urología. Ene Ediciones. Madrid. Mayo 1996.

Dr. O. Rodríguez Faba

(Trabajo recibido el 4 de octubre 2004) 